\title{
Study on biosynthesis of ginsenosides in the leaf of Panax ginseng by seasonal flux analysis
}

\author{
Dongmin $\mathrm{Kim}^{1} \cdot$ Jaehong Han ${ }^{1}$
}

Received: 17 August 2019 / Accepted: 10 September 2019 / Published Online: 31 December 2019

(C) The Korean Society for Applied Biological Chemistry 2019

\begin{abstract}
Seasonal ginsenoside flux in the leaves of 5-year-old Panax ginseng was analyzed from the field-grown ginseng, for the first time, to study possible biosynthesis and translocation of ginsenosides. The concentrations of nine major ginsenosides, $\mathrm{Rg} 1$, $\mathrm{Re}, \mathrm{Rh} 1, \mathrm{Rg} 2, \mathrm{R}-\mathrm{Rh} 1, \mathrm{Rb} 1, \mathrm{Rc}, \mathrm{Rb} 2$, and Rd, were determined by UHPLC during the growth in between April and November. It was confirmed total ginsenoside content in the dried ginseng leaves was much higher than the roots by several folds whereas the composition of ginsenosides was different from the roots. The ginsenoside flux was affected by ginseng growth. It quickly increased to $10.99 \pm 0.15$ (dry wt\%) in April and dropped to $6.41 \pm 0.14 \%$ in May. Then, it slowly increased to $9.71 \pm 0.14 \%$ in August and maintained until October. Ginsenoside Re was most abundant in the leaf of $P$. ginseng, followed by $\mathrm{Rd}$ and $\mathrm{Rg} 1$. Ginsenosides Rf and Ro were not detected from the leaf. When compared to the previously reported root data, ginsenosides in the leaf appeared to be translocated to the root, especially in the early vegetative stage even though the metabolite translocated cannot be specified. The flux of ginsenoside R-Rhl was similar to the other (20S)-PPT ginsenosides. When the compositional changes of each ginsenoside in the leaf was analyzed, complementary relationship was observed from ginsenoside $\mathrm{Rg} 1$ and $\mathrm{Re}$, as well as from ginsenoside $\mathrm{Rd}$ and $\mathrm{Rb} 1+\mathrm{Rc}$. Accordingly, ginsenoside $\mathrm{Re}$ in the leaf was proposed to be synthesized from ginsenoside Rg1. Similarly, ginsenosides Rb1 and Rc were proposed to be synthesized from Rd.
\end{abstract}

Jaehong Han $(\bowtie)$

E-mail: jaehongh@cau.ac.kr

${ }^{1}$ Metalloenzyme Research Group and Department of Plant Science and Technology, Chung-Ang University, Anseong 17546, Republic of Korea

This is an Open Access article distributed under the terms of the Creative Commons Attribution Non-Commercial License (http://creativecommons. org/licenses/by-nc/3.0/) which permits unrestricted non-commercial use, distribution, and reproduction in any medium, provided the original work is properly cited.
Keywords Biosynthesis $\cdot$ Ginsenosides $\cdot$ Panax ginseng $\cdot$ Seasonal flux $\cdot$ Translocation $\cdot$ UHPLC

\section{Introduction}

Continuous analysis of secondary metabolites through the plant growth stages provides valuable data, even though it is not an easy task when the samples collected from cultivation field [1]. As a powerful modeling tool, plant metabolite flux can also lead to the biosynthetic pathway elucidation of plant secondary metabolites. In principle, long-term flux change of biosynthetically-related secondary metabolites reflects the changes of the corresponding enzymes activity resulting from the regulation of different gene expression [2]. In the case of ginsenosides, a group of bioactive secondary metabolites in ginseng is presumably biosynthesized from the sequential glycosylation of protopanaxadiol (PPD) and protopanaxatriol (PPT). But, each structurally related ginsenoside has different biological activities. The unique situation of ginsenosides makes metabolic flux investigation meaningful, even without isotope-labelling.

Recently, we have reported seasonal ginsenoside flux change in the root of field-grown Panax ginseng to assess this proposition [3]. Apart from the practical finding that the highest ginsenoside content was produced in May not in the harvest season, we have also confirmed that the seasonal ginsenoside flux in the root could be utilized for the biosynthetic pathway elucidation. Encouraged by the results, seasonal variation of ginsenosides in the ginseng leaf was investigated with the same ginseng samples of which roots were analyzed previously (Fig. 1). This study is expected to not only elucidate the ginsenosides biosynthetic pathway in the leaf, but also insinuate translocation of ginsenosides between ginseng leaf and root.

In 2017, more than 23,310 tons of ginseng were harvested in Korea [4], and more than thousands of tons of ginseng leaves 


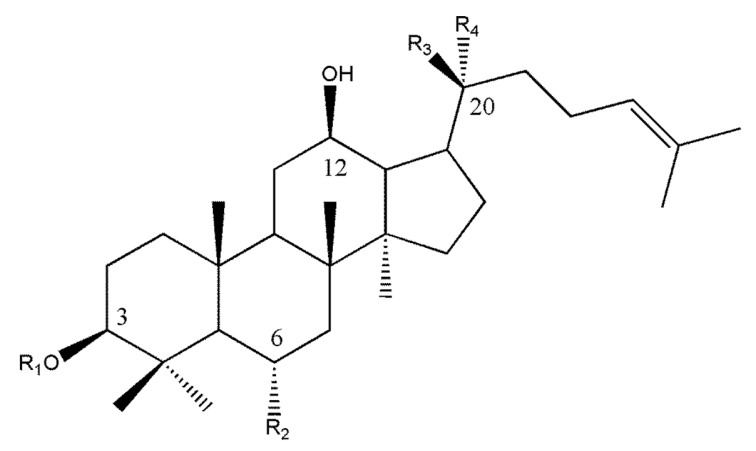

\begin{tabular}{ccccc}
\hline Ginsenoside & R1 & R2 & R3 & R4 \\
\hline $\mathrm{Rg} 1$ & $-\mathrm{H}$ & $-\mathrm{O}-\mathrm{Glc}$ & $-\mathrm{O}-\mathrm{Glc}$ & $-\mathrm{CH}_{3}$ \\
\hline $\mathrm{Re}$ & $-\mathrm{H}$ & $-\mathrm{O}-\mathrm{Glc}^{2}-{ }^{1} \mathrm{Rha}$ & $-\mathrm{O}-\mathrm{Glc}$ & $-\mathrm{CH}_{3}$ \\
\hline $\mathrm{Rh} 1$ & $-\mathrm{H}$ & $-\mathrm{O}-\mathrm{Glc}$ & $-\mathrm{OH}$ & $-\mathrm{CH}_{3}$ \\
\hline $\mathrm{Rg} 2$ & $-\mathrm{H}$ & $-\mathrm{O}-\mathrm{Glc}^{2}-{ }^{1} \mathrm{Rha}$ & $-\mathrm{OH}$ & $-\mathrm{CH}_{3}$ \\
\hline$(20 \mathrm{R})-\mathrm{Rh} 1$ & $-\mathrm{H}$ & $-\mathrm{O}-\mathrm{Glc}$ & $-\mathrm{CH}_{3}$ & $-\mathrm{OH}$ \\
\hline $\mathrm{Rb} 1$ & $-\mathrm{Glc}^{2}-{ }^{1} \mathrm{Glc}$ & $-\mathrm{H}$ & $-\mathrm{O}-\mathrm{Glc}^{6}-{ }^{1} \mathrm{Glc}$ & $-\mathrm{CH}_{3}$ \\
\hline $\mathrm{Rc}$ & $-\mathrm{Glc}^{2}-{ }^{1} \mathrm{Glc}$ & $-\mathrm{H}$ & $-O-\mathrm{Glc}^{6}-{ }^{1} \mathrm{Ara}(\mathrm{f})$ & $-\mathrm{CH}_{3}$ \\
\hline $\mathrm{Rb} 2$ & $-\mathrm{Glc}^{2}-{ }^{1} \mathrm{Glc}$ & $-\mathrm{H}$ & $-O-\mathrm{Glc}^{6}-{ }^{1} \mathrm{Ara}(\mathrm{p})$ & $-\mathrm{CH}_{3}$ \\
\hline $\mathrm{Rd}$ & $-\mathrm{Glc}^{2}-{ }^{1} \mathrm{Glc}$ & $-\mathrm{H}$ & $-O-\mathrm{Glc}$ & $-\mathrm{CH}_{3}$
\end{tabular}

Fig. 1 Molecular structures of ginsenosides analyzed from ginseng leaf

were wasted. Due to high content of ginsenosides, the leaf of $P$. ginseng has been recognized as a promising bioactive material exhibiting anti-fatigue, hypoglycemic, anti-obesity, and anticancer activities [5-7]. Although leaves of $P$. ginseng cultivated in Korea has a great potential for the bio-related industry as an alternative of radix ginseng [8], extensive study on the ginsenosides flux is very limited and most studies on ginseng leaf were performed at the vegetative stage.

On the other hand, ginseng leaf harvest is practically only possible at the harvest season, because earlier leaf amputation inhibits ginseng growth. Hence, leaf ginsenoside flux changes through the growth stages will be necessary to overcome the obstacles hindering biotechnological applications of ginseng leaf as a new functional bioactive material. This is the first extensive study on the seasonal ginsenoside flux in the leaf of the fieldcultivated 5-year Korean ginseng.

\section{Materials and Methods}

Ginsenoside F1, F3, Ra1, Rg1, Re, Rf, Rh1, Rg2, Rg3, Ro, (20R)$\mathrm{Rh} 1, \mathrm{Rb} 1, \mathrm{Rc}, \mathrm{Rb} 2$, and $\mathrm{Rd}$ were purchased from Embo Laboratory (Daejeon, Korea) in Korea and used for reference compounds. Voucher specimen of ginseng leaves harvested throughout the year 2017 were obtained from Phytobean AC. LTD (Yecheon-gun, Korea). HPLC-grade methanol (MeOH), water and acetonitrile $(\mathrm{MeCN})$ were purchased from Honeywell
(Morristown, NJ, USA).

\section{Ginseng samples and analyte preparation}

The leaves of $P$. ginseng from 20 ginseng plants were collected from the cultivation field at Bongwha, Korea $\left(36^{\circ} 85^{\prime} 2^{\prime \prime N}\right.$; $128^{\circ} 80^{\prime} 63^{\prime \prime} \mathrm{E}$; altitude $280 \mathrm{~m}$ ) in 2017 . The leaves were cut at the base near petiole, and dried under shade at room temperature until no changes of weight were observed. The dried leaves were brittle and pulverized by blender. The average dry weight was about $10 \%$ of fresh ginseng leaves. For the analysis of ginsenosides by UHPLC (Ultimate 3000, ThermoFisher, Waltham, MA, USA), $50.0 \mathrm{mg}$ of dried powder were added to the $10.0 \mathrm{~mL}$ of $80 \%$ methanol, and the solution was sonicated with $135 \mathrm{kHz}$ for $2 \mathrm{~h}$ at $40{ }^{\circ} \mathrm{C}$ (5510R-DTH waterbath sonicator, Bransonic, Danbury, CT, USA). The solution was centrifuged for $15 \mathrm{~min}(2000 \times \mathrm{g})$ and the supernatant $(8.0 \mathrm{~mL})$ was transferred to $30 \mathrm{~mL}$ glass vial in the Micro-Cenvac (NB-503CIR, N-Biotek, Bucheon, Korea). After dryness for $2 \mathrm{~h}$ at $60{ }^{\circ} \mathrm{C}$, the residue was dissolved in $10.0 \mathrm{~mL}$ of methanol. About $3 \mathrm{~mL}$ of methanolic solution was filtered through a $0.22-\mu \mathrm{m}$ cellulose filter to prepare the UHPLC analyte.

\section{General UHPLC Method}

A Thermo Ultimate 3000 equipped with a PDA detector and a C18 Phenomenex Kinetex ${ }^{\mathrm{TM}}$ (Torrance, CA, USA) column $(2.1 \times 100 \mathrm{~nm}, 1.7 \mu \mathrm{m})$, was used for UHPLC analysis. The injection volume was $2 \mu \mathrm{L}$ and the flow rate was $0.6 \mathrm{~mL} / \mathrm{min}$. The mobile phase for the analysis of ginsenosides was composed of $0.001 \%$ phosphoric acid in deionized water (solvent A) and $0.001 \%$ phosphoric acid in MeCN (solvent B). For the eluent gradient system, solution B started at $18 \%$ for $5 \mathrm{~min}$ and increased linearly to $45 \%$ from $5 \mathrm{~min}$ to $25 \mathrm{~min}$, to $90 \%$ from $25 \mathrm{~min}$ to 30 min, and was held constant at $90 \%$ for 2 min before returning to the initial composition. The column was maintained at $40{ }^{\circ} \mathrm{C}$. The chromatograms were generated by $203 \mathrm{~nm}$ UV absorption.

\section{Calibration Curves of Ginsenosides}

The standard solutions of ginsenosides were diluted to five to nine different concentrations between 1 and $100 \mu \mathrm{g} / \mathrm{mL}$, depending on the abundance in the ginseng sample. The analytes were then analyzed by UHPLC in triplicate, and the calibration curves were constructed by plotting peak area versus the concentration of ginsenoside (Supplementary material, Table S1).

\section{Results}

\section{Ginsenosides in the leaf of field-cultivated $\boldsymbol{P}$. ginseng}

The ginseng leaves collected biweekly in between April and November 2017 from Bongwha, Korea (Supplementary material, Fig. S1) were analyzed by UHPLC (Fig. S2). In the area where the samples collected from, ginseng started to bud in the early April and grew until August. Necrosis of the leaves began in the 


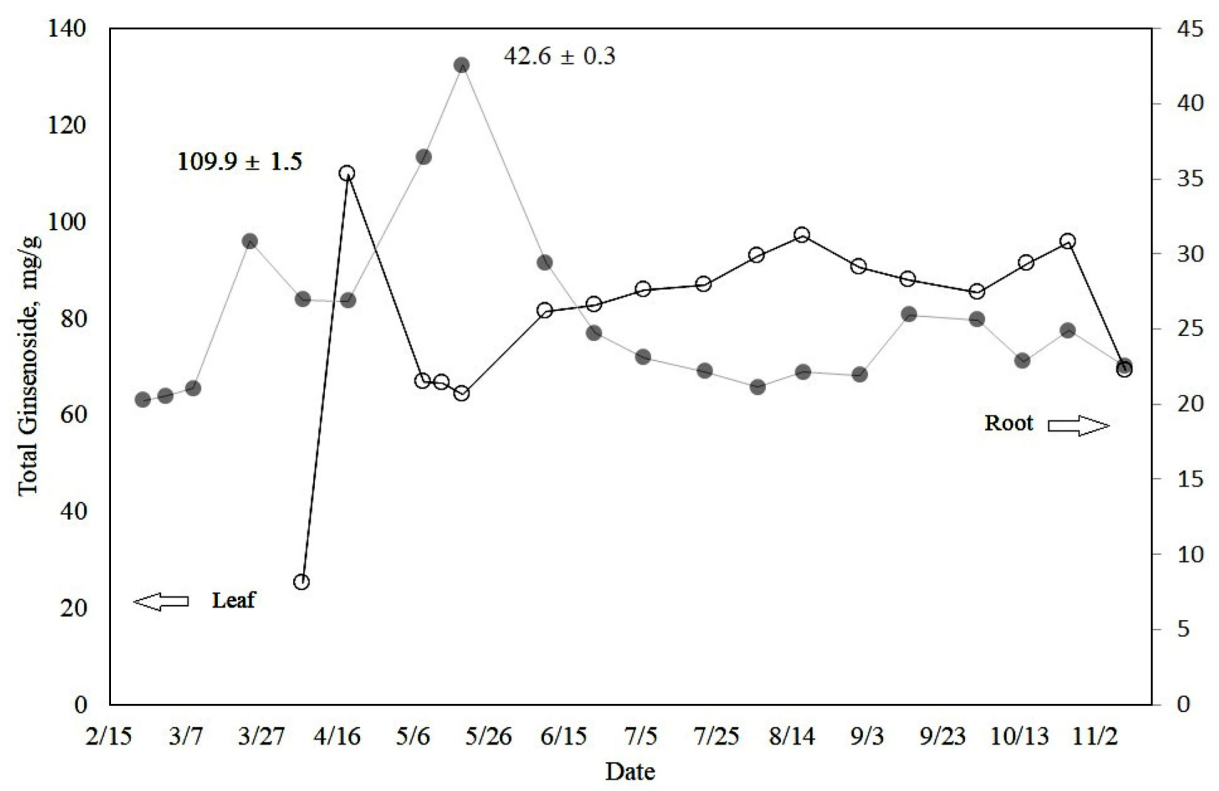

Fig. 2 Total ginsenoside flux ( $\mathrm{mg} / \mathrm{g}$ in dry weight) changes of ginseng leaf and root. The root data was obtained from ref [3]

beginning of September due to temperate climate.

Under the optimized UHPLC conditions that resolve major ginsenosides, the chromatographic separation of Rh1 and Rg2 was not complete. However, ginsenoside Rg2 was minor component and other major ginsenosides could not be resolved with the solvent systems that separate Rh1 and Rg2. Therefore, vertical integration was adopted for the each ginsenoside. Likewise, the concentration of ginsenoside Rc, eluted with ginsenoside F1, was obtained by the vertical integration, too. Along with other major ginsenosides, the presence of ginsenoside (20R)-Rh1 (R-Rh1) in the leaf was confirmed, that was distinctive from all the chromatograms [9]. Interestingly, ginsenosides Rf and Ro characteristic in the ginseng root were not detected from the ginseng leaf samples, even though ginsenoside $\mathrm{Rf}$ from the leaf was reported previously [10]. The literature inconsistency of ginsenoside Rf existance in the leaf could be in part due to the different biosynthetic gene expression upon the cultivation years [11].

\section{Seasonal ginsenoside flux in the leaf of $P$. ginseng}

In the early vegetative stage (April), the contents of ginsenosides quickly increased with the formation of leaves. After the quick drop in May, total ginsenoside steadily increased from June until November (Fig. 2). The abrupt decrease of leaf ginsenosides could be related with translocation to the root. The highest total ginsenoside content $(109.9 \pm 1.5 \mathrm{mg} / \mathrm{g} \mathrm{DW})$, equal to $11 \%$ of dried leaf, was found in the middle of April, but total ginsenosides in the leaves stayed steady at around $90 \mathrm{mg} / \mathrm{g}$ in between July and October. In November, the total ginsenoside content decreased to $69.2 \pm 1.4 \mathrm{mg} / \mathrm{g}$ with leaf senescence. The total ginsenoside content of the fallen leaves collected in December was measured about $3 \%$ of total mass (data not shown).
When each ginsenoside was compared, ginsenoside $\mathrm{Re}, \mathrm{Rd}$, and Rg1 were major ginsenosides in the leaf (Fig. 3, Table S2). Interestingly, ginsenoside $\mathrm{Re}$ and $\mathrm{Rg} 1$, but $\mathrm{Rd}$, were major in the leaves of 5-year-old $P$. ginseng cultivated in China [12]. According to Liu et al., ginsenoside $\mathrm{Rb} 3$, Rc and notoginsenoside FC were reported as major ginsenosides of $P$. notoginseng leaf [13]. Contrary to ginseng root in which PPD is more abundant by 2.5 times [3], the ginseng leaf showed high content of PPT type ginsenosides. The ratio of PPD/PPT $\leq 1$ was observed, except Apr 19, and gradually decreased through the year (Fig. 4). Therefore, it was found that PPT biosynthesis is more active in ginseng leaf.

Among PPT group, the concentration of ginsenosides always followed the order of $\operatorname{Re}>\operatorname{Rg} 1>\mathrm{R}-\mathrm{Rh} 1>\mathrm{Rh} 1>\mathrm{Rg} 2$, with the exception of the first measurement on Apr 7. Exception of R-Rh1 of which the absolute configuration on $C-20$ is $R$, the other PPT ginsenosides, Re, Rg1, Rh1, and Rg2, have $S$-configuration on 20$C$. When the stereoisomers were compared, the ratio of (20S)-PPT ginsenosides $(S$-PPT) and R-Rh1 was closed to 10 with exception of the first data on Apr 7 (Fig. S3). Therefore, it was proposed that the biosynthesis of R-Rh1 is correlated to S-PPT either enzymatically or non-enzymatically.

As ginseng grew, ginsenoside Rc decreased and ginsenoside $\mathrm{Rb} 1$ increased. However, relative distribution of PPD type ginsenosides in the leaf was generally found as $\mathrm{Rd}>\mathrm{Rb} 2 \approx \mathrm{Rc} \approx$ $\mathrm{Rb} 1$ throughout the year (Fig. 3). The flux of ginsenoside Rb2 didn't change much. Interestingly, the most abundant root PPT ginsenoside was reported as Rb1, followed by Rc [3]. Therefore, it is strongly suggested that glycosylation of ginsenoside $\mathrm{Rd}$ is less active in ginseng leaf.

To investigate possible biosynthetic pathway of leaf ginsenosides, fluxes of ginsenoside composition in PPT and PPD groups were 


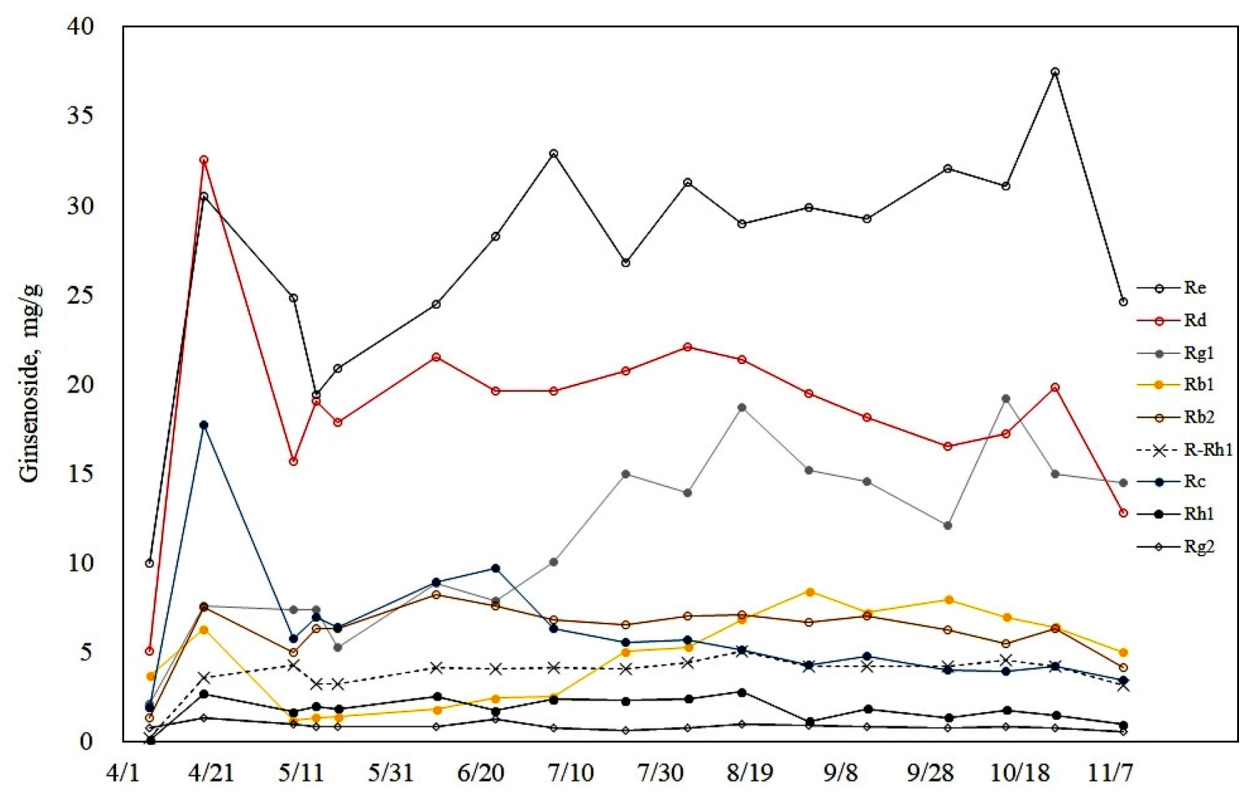

Fig. 3 Ginsenosides flux in the leaf of five-year ginseng throughout the year

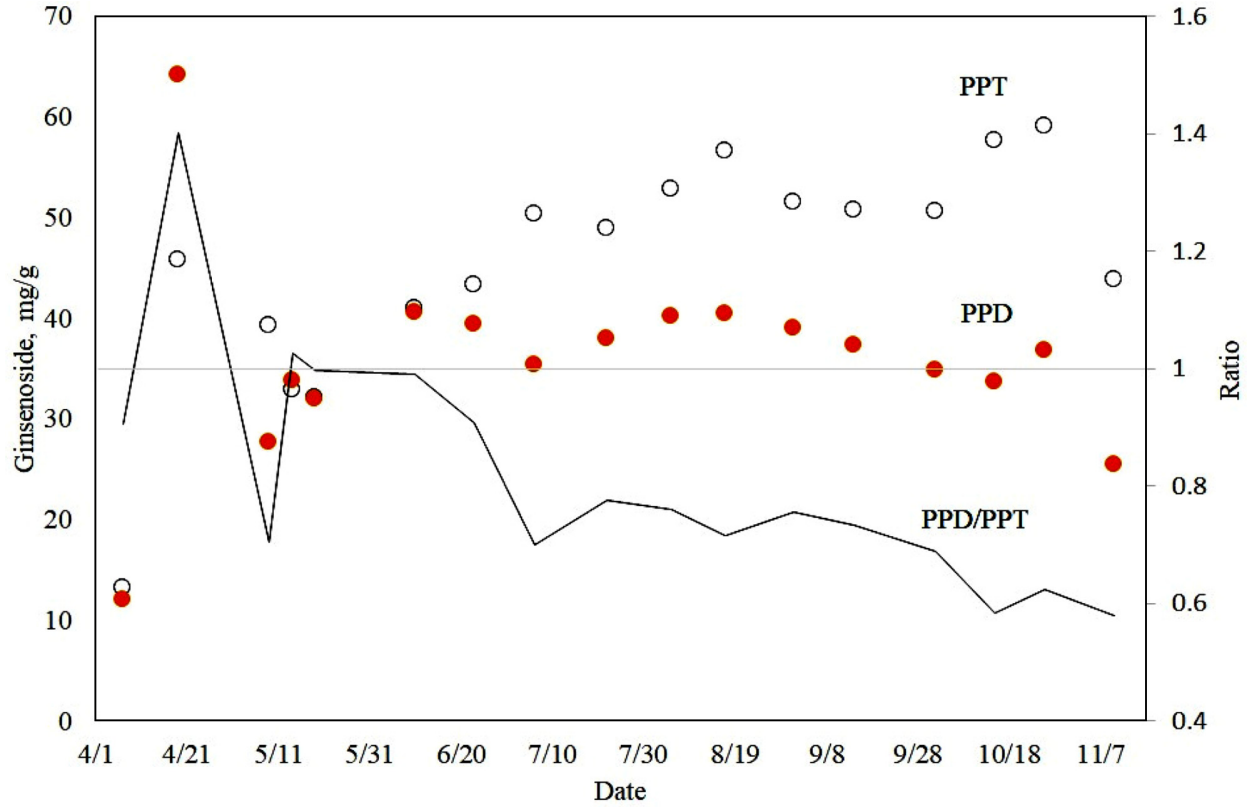

Fig. 4 The flux of PPT and PPD ginsenosides and the ratio of PPD/PPT (line) in the leaf of five-year ginseng. The scales of PPD/PPT ratio are represented at the right $\mathrm{Y}$-axis

analyzed. In the PPT group, percentile flux of each ginsenoside, $\mathrm{Rg} 1, \mathrm{Re}, \mathrm{Rh} 1$, and Rg2, in S-PPT ginsenosides was obtained. The percentage of the most abundant ginsenoside Re slowly decreased from 77 to $61 \%$ throughout the year. On the contrary, the second major PPT ginsenoside, $\mathrm{Rg} 1$, slowly increased from 16 to $36 \%$. Both ginsenosides consituted more than $90 \%$ of total S-PPT and steadily increased to $96 \%$ as ginseng grew. Interestingly, the percentile fluxes of ginsenoside $\mathrm{Rg} 1$ and $\mathrm{Re}$ in the growth period were mirror image each other (Fig. 5A). Therefore, it was concluded that ginsenoside $\operatorname{Rg} 1$ and $\mathrm{Re}$ are the final PPT metabolites in ginseng leaf. The activity of the putative $\mathrm{Rg} 1$ rhamnose transferase seems to determine the ratio of $\operatorname{Rg} 1$ and $\mathrm{Re}$. The contents of Rg2 and Rh1 were very low through the year, and which implies the putative Rhl glucose transferase is very active in the leaf.

Percentile flux of each PPD ginsenoside could not be simply correlated (Fig. 5B). After careful simulation, it was found that percentile fluxes of ginsenoside $\mathrm{Rd}$ and $\mathrm{Rb} 1+\mathrm{Rc}$ formed mirror 

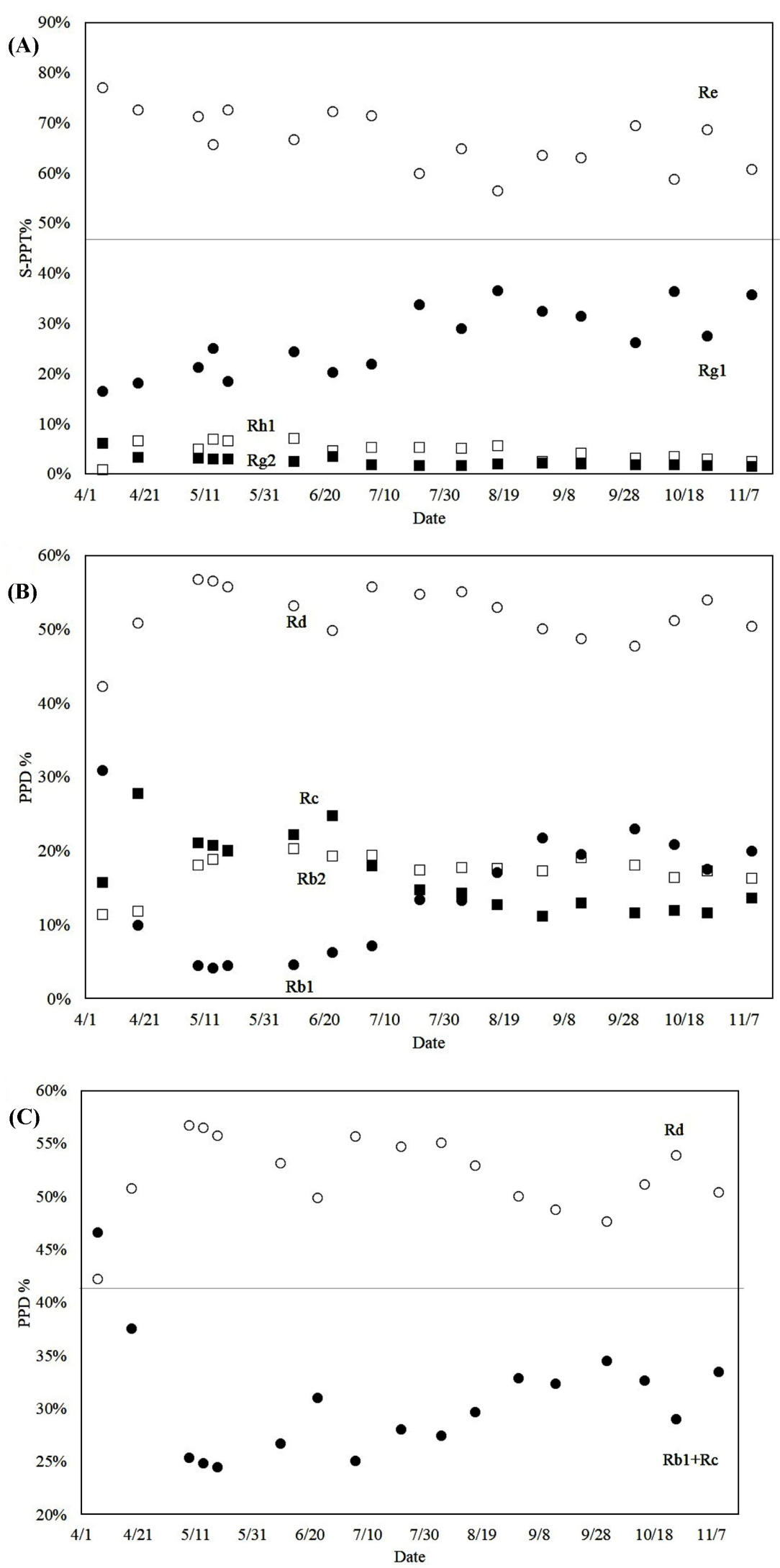

Fig. 5 Percentile flux of S-PPT (A) and PPD (B), as well as percentile fluxes of ginsenosides Rd and the sum of ginsenosides Rb1 and Rc (C) 


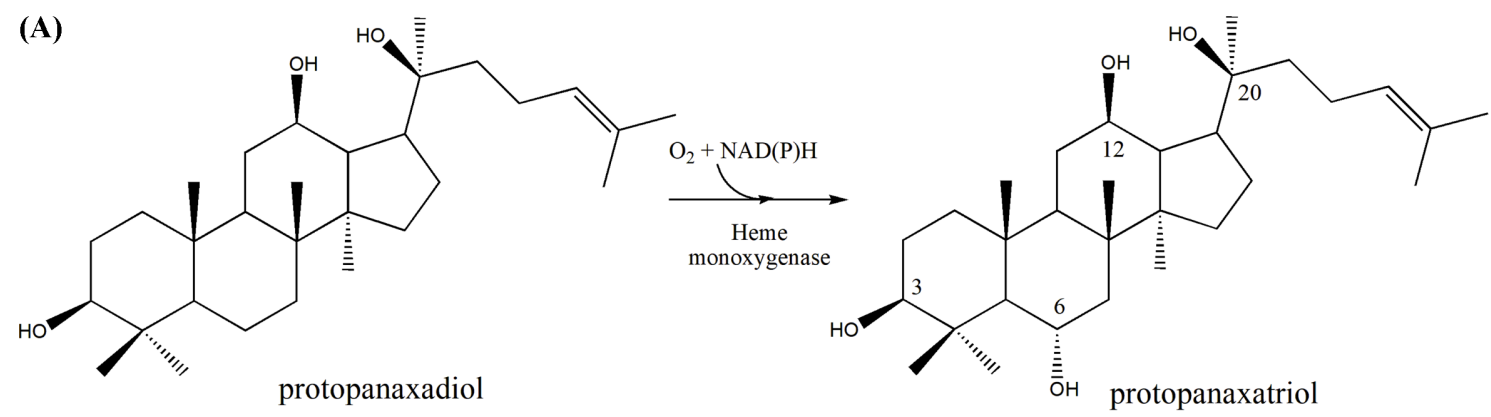

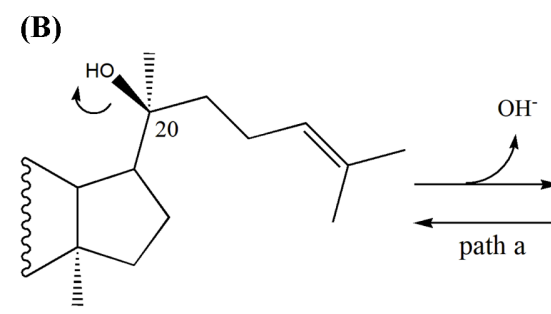

(20S)-PPT

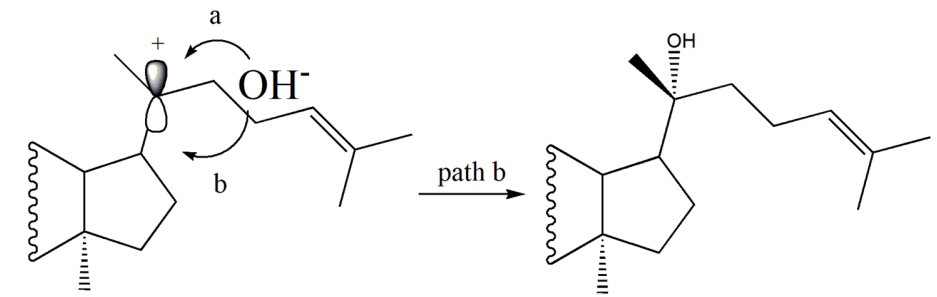

(20R)-PPT

Fig. 6 Plausible mechanism of protopanaxatriol biosynthesis (A) and the racemization of (20S)-PPT. Protopanaxatriol synthesis is not related to the racemization on $C-20$ (A), and glucosidation on $20-\mathrm{OH}$ will prevent the racemization on $C-20$ (B)

image as shown in Fig. 5C. Therefore, ginsenoside Rd was proposed as a common biosynthetic precursor of ginsenoside $\mathrm{Rb} 1$ and $\mathrm{Rc}$ in ginseng leaf.

\section{Discussion}

Leaf of $P$. ginseng has a great potential for the application of bioactive natural product. However, study on ginsenosides in the leaf is scarcer than the root, and the results are very diverse. For examples, Rh1 and Rb3 were reported as the most abundant ginsenosides in the leaf of 6-year-old $P$. ginseng (Chunpoong cultivar) grown in Korea [14]. Besides, Ro and Rf were consistently found in significant amounts from the leaf of $P$. ginseng grown in China [12]. It is estimated that different sampling time of ginseng leaves would have resulted in different results. In this work, we have investigated seasonal ginsenoside flux in the leaf of 5-year-old field grown $P$. ginseng. The results are expected to provide extensive data on ginsenoside compositional changes during the growth period, as well as biosynthetic pathway of leaf ginsenosides through the metabolic flux analysis. In this work, both diastereomers of ginsenoside Rh1, (20S)-Rh1 and $(20 R)$-Rh1, were also analyzed to investigate the origin of $(20 R)$ ginsenosides.

The UHPLC analysis of ginseng leaves did not detect ginsenoside Rf and Ro (Fig. S1), similar to the leaves of hydroponically grown ginseng [15]. The dried leaf samples showed high contents of total ginsenoside (Fig. 2), even more than ginseng roots [3]. However, it should be mentioned that total ginsenosides content of leaf and root were similar when fresh samples were compared. When two data were compared in a graph, the decrease of leaf ginsenosides in May was correlated clearly to the increase of root ginsenosides (Fig. 2). Therefore, ginsenoside translocation from leaf to root was suggested. Even though translocation of ginsenosides from the leaf to the flower is possible, it was ruled out because flowers are removed in the conventional ginseng cultivation practice.

The ginsenoside composition in the leaf was different among different Panax species. Ginsenoside Re was the most abundant ginsenoside in the leaf of $P$. ginseng, whereas $\mathrm{Rb} 3$ was most abundant in $P$. notoginseng and P. quinquefolius $[13,16]$. When the ginsenoside composition in the leaf was compared to the root samples, interesting point was found. The abundancy of $\operatorname{Re}>\mathrm{Rd}$ $>\operatorname{Rg} 1$ was found in the leaf, whereas the order of $R b 1>R c>R g 1$ was found in the root of ginseng [3]. It seems transglycosylation of PPT type $\mathrm{Re}$ is more active in ginseng leaf while transglycosylation of PPD type $\mathrm{Rd}$ is more active in ginseng root.

Biosynthesis of metabolites in ginseng is known to be tissue specific. For example, polyacetylenes exhibiting important biological activity are only found from ginseng root [17]. Therefore, it is strongly suggested the activity of the enzymes involved in ginsenoside biosynthesis is quite different between leaf and root. The ratio of PPD/PPT in ginseng leaf was found at the range of 0.6 and 1.4 , very different from the root (PPD/PPT $\approx 2.5$ ), throughout the year (Fig. 4). The high contents of PPT ginsenosides in the leaf are probably due to the more available molecular oxygen which is the other substrate of CYP type monoxygenase involved in the conversion of PPD to PPT [18] (Fig. 6A). It was also found that flux change of ginsenoside R-Rh1 was related to that of S-PPT 


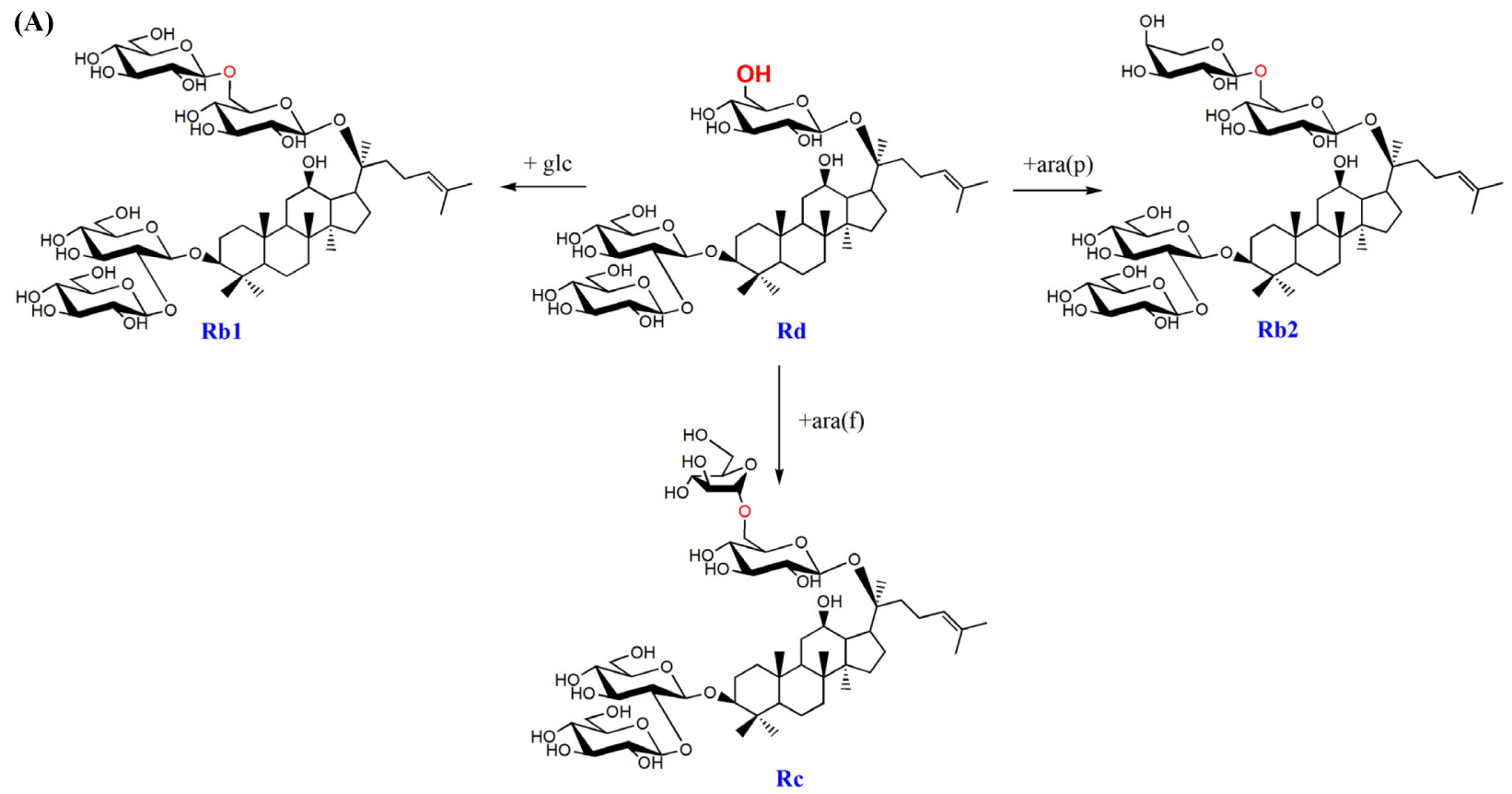

(B)

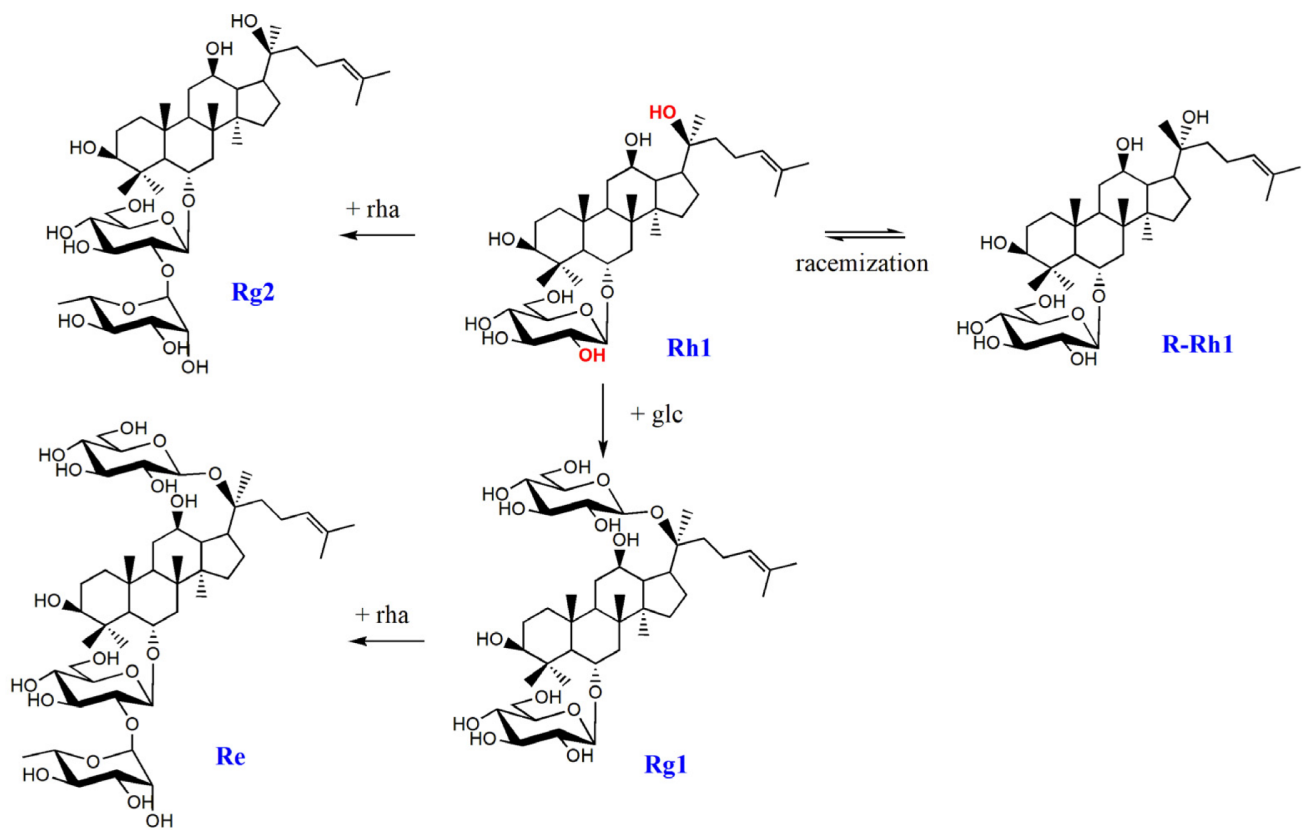

Fig. 7 Possible biosynthetic pathway for the selected PPD (A) and PPT (B) in the leaf of $P$. ginseng

ginsenosides, and the ratios of S-PPT/R-Rh1 were found at around 10 throughout the year, except the first data on Apr 7 (Fig. S3). The occurrence of $(20 R)$-ginsenoside stereoisomers, such as R$\mathrm{Rh} 1$, is considered due to the intrinsic lability of tertiary alcohol at $C-20$ which facilitates tertiary carbocation formation (Fig. 6B). Since $C-20$ is a labile tertiary alcohol closed to equatorial $12-\mathrm{OH}$, the isomerization between ginsenoside Rhl and R-Rhl is considered feasible non-enzymatically. At the same time, direct glucosidation at 6-OH of PPT, resulting in both Rh1 and R-Rh1, cannot be ruled out. However, following biosynthetic steps of $20 O$-glucosidation will prohibit such racemization.

The complementary relationship of certain ginsenosides in PPT and PPD groups was found as mirror images of percentile flux as shown at Fig. 5A and $\mathrm{C}$, respectively. In ginseng leaf, metabolic fluxes of $\mathrm{Rd}$ and $\mathrm{Rb} 1+\mathrm{Rc}$ was suggested to use the same PPD metabolic pool (Fig. 5C) while that of Rb2 didn't show any correlation with other PPD ginsenosides. The simplest interpretation would be that the glycosylation of Rd catalyzed by the enzyme 
which utilize glucosyl and arabiofuranosyl groups equally (Fig. 7A). It is also suggested that different enzyme involves in the Rb2 synthesis, and the catalysis is relatively slow compared to the biosynthesis of Rbl and Rc. In PPT biosynthesis, it was clear that metabolic fluxes of Re and $\operatorname{Rg} 1$ were related and the transfer of rhamnosyl group to Rg1 would produce Re. The glucose transfer at the $20-\mathrm{OH}$ of Rh1 seemed to be faster than the racemization of Rh1 or the arabinose transfer (Fig. 7B). Because fluxes of Re and $\mathrm{Rg} 2$ cannot correlated in any ways, it is proposed that different enzymes catalyze the rhamnose transfer to $\mathrm{Rg} 1$ and Rh1, respectively.

In conclusion, major ginsenosides flux was measured in the leaf of 5-year-old field-grown $P$. ginseng by UHPLC. The high content of ginsenosides in the leaf proved that it can be a potential bioactive material for the industrial applications. The changes of total ginsenoside content strongly implied the possible translocation from leaf to root, but the flux change alone could not specify the specific metabolite being translocated. Percentile fluxes of PPD and PPT ginsenosides suggested that the synthesis of Rb1 and Rc may be catalyzed by the same enzyme and the synthesis of $\operatorname{Rg} 2$ and Re may be catalyzed by different enzymes.

It was confirmed that the seasonal flux changes of structurally related metabolites could provide valuable information for the investigation of biosynthetic pathway. Future biochemical study on the specific ginsenoside biosynthetic metabolism would confirm the conclusions.

Acknowledgments This work was supported by Korea Institute of Planning and Evaluation for Technology in Food, Agriculture, Forestry and Fisheries (IPET) through the Science and Technology-Based Creative Agriculture Promotion Priority Project, funded by Ministry of Agriculture, Food and Rural Affairs (MAFRA) (514006032HD060).

\section{References}

1. Bacher A, Rieder C, Eichinger D, Arigoni D, Fuchs G, Eisenreich W (1998) Elucidation of novel biosynthetic pathways and metabolite flux patterns by retrobiosynthetic NMR analysis. FEMS Microbiol Rev 22: $567-598$

2. Baghalian K, Hajirezaei MR, Schreiber F (2014) Plant metabolic modeling: achieving new insight into metabolism and metabolic engineering. Plant Cell 26: 3847-3866

3. Kim D, Kim M, Rana G, Han J (2018) Seasonal Variation and Possible Biosynthetic Pathway of Ginsenosides in Korean Ginseng Panax ginseng Meyer. Molecules 23: 1824

4. Ministry of Agriculture, Food and Rural Affairs (2018) 2017 Ginseng Statistics, Sejong

5. Mao Q, Bai M, Xu JD, King M, Zhu LY, Zhu H, Wang Q, Li SL (2014) Discrimination of leaves of Panax ginseng and $P$. quinquefolius by ultra high performance liquid chromatography quadrupole/time-of-flight mass spectrometry based metabolomics approach. J Pharmaceut Biom Anal 97: $129-140$

6. Wang H, Peng D, Xie J (2009) Ginseng Leaf-Stem: Bioactive Constituents and Pharmacological Functions. Chin Med 4: 20

7. Zhang Y, Zhang J, Liu C, Yu M, Li S (2017) Extraction, Isolation, and Aromatase Inhibitory Evaluation of Low-Polar Ginsenosides from Panax ginseng Leaves. J Chromatogr A 1483: 20-29

8. Shin BK, Park HY, Han J (2010) Enzymatic Biotransformation of Red Ginseng and the Compositional Change of Ginsenosides. J Korean Soc Appl Biol Chem 53: 533-538

9. Lee J, Choi BR, Kim YC, Choi D, Lee YS, Kim GS, Baek NI, Lee DY (2017) Comprehensive Profiling and Quantification of Ginsenosides in the Root, Stem, Leaf, and Berry of Panax ginseng by UPLC-QTOF/MS. Molecules 22: 2147

10. Yang SO, Lee SW, Kim YO, Sohn SH, Kim YC, Hyun DY, Hong YP, Shin YS (2013) HPLC-based metabolic profiling and quality control of leaves of different Panax species. J Ginseng Res 37: 248-253

11. Kim YJ, Joo SC, Shi J, Hu J, Sukweenadhi J, Mohanan P, Yang DC, Zhang D (2018) Metabolic dynamics and physiological adaptation of Panax ginseng during development. Plant Cell Rep 37: 393-410

12. Zhang YC, Li G, Jiang C, Yang B, Yang HJ, Xu HY, Huang LQ (2014) Tissue-specific distribution of ginsenosides in different aged ginseng and antioxidant activity of ginseng leaf. Molecules 19: 17381-17399

13. Liu F, Ma N, He C, Hu Y, Li P, Chen M, Su H, Wan JB (2018) Qualitative and quantitative analysis of the saponins in Panax notoginseng leaves using ultra-performance liquid chromatography coupled with time-of-flight tandem mass spectrometry and high performance liqu id chromatography coupled with UV detector. J Ginseng Res 42: 149-157

14. Kang OJ, Kim JS (2016) Comparison of ginsenoside contents in different parts of Korean ginseng (Panax ginseng C.A. Meyer). Prev Nutr Food Sci 21: 389-392

15. Kim YJ, Jeon JN, Jang MG, Oh JY, Kwon WS, Jung SK, Yang DC (2014) Ginsenoside profiles and related gene expression during foliation in Panax ginseng Meyer. J Ginseng Res 38: 66-72

16. Wang CZ, Wu JA, McEntee E, Yuan CS (2006) Saponins composition in American ginseng leaf and berry assayed by high-performance liquid chromatography. J Agric Food Chem 54: 2261-2266

17. Woo HC, Shin BK, Cho I, Koo H, Kim M, Han J (2011) Anti-obesity Effect of Carbon Dioxide Supercritical Fluid Extracts of Panax ginseng C. A. Meyer. J Korean Soc Appl Biol Chem 54: 738-743

18. Han JY, Hwang HS, Choi SW, Kim HJ, Choi YE (2012) Cytochrome P450 CYP716A53v2 Catalyzes the Formation of Protopanaxatriol from Protopanaxadiol During Ginsenoside Biosynthesis in Panax Ginseng. Plant Cell Physiol 53: 1535-1545 